Journal of Mathematics and Informatics

Vol. 10, 2017, 95-101

ISSN: 2349-0632 (P), 2349-0640 (online)

Published 11 December 2017

www.researchmathsci.org

DOI: http://dx.doi.org/10.22457/jmi.v10a13

Journal of

Mathematics and

Informatics

\title{
Connections between Cylinder, Frustum of a Cone with Truncated Octahedral Number and Other Special Numbers
}

\author{
G.Janaki and P.Saranya \\ Department of Mathematics, Cauvery College for Women \\ Trichy-18, Tamilnadu, India \\ Received 1 November 2017; accepted 2 December 2017
}

Abstract. In this paper, we find the relation among the geometrical figures cylinder and frustum of the cone with truncated octahedral number and some special numbers such as gnomonic number and polygonal numbers.

Keywords: Frustum of the cone, Lateral surface area of the cylinder, Truncated Octahedral number

\section{AMS Mathematics Subject Classification (2010): 11D09}

\section{Introduction}

The systematic study of number theory was initiated around 300 B.C. Over a thousand years later around 972 A.D. Arab mathematicians formulated the congruent number problem to decide whether or not a given positive integer $\mathrm{n}$ is area of right triangle, all three of whose sides are rationals. Another 1000 years later in 1976 Diffie and Hellman introduced the first ever public-key cryptosystem which enabled to communicate secretly over public communication channels. By then there has been a rapid growth of inventions and the research continues.

For various ideas and problems one may refer [1-5].Various relations between Pythagorean triangles and special numbers are studied in [6-16].Relations between Rectangles and special numbers are studied in [17-18].

Recently in [19] some observations regarding Truncated Octahedral number and in [20] Correlation of cylinder and frustum of the cone with special numbers are recorded.

In this paper, we find the relation among the geometrical figures cylinder and frustum of the cone with truncated octahedral number and some special numbers such as gnomonic number and polygonal numbers.

\section{Method of analysis}

Let $\mathrm{r}, \mathrm{h}$ be the base radius and height of the cylinder respectively. Suppose a frustum of the cone with the same height ' $h$ ' of the cylinder and the circular end at the bottom of this frustum coincides with the base of the cylinder is attached on either of its base. 


\section{G.Janaki and P.Saranya}

This means that the base radius of the cylinder is equal to the radius of the circular end at the bottom of the frustum. Let $\mathrm{R}$ be the radius of the circular end at the top of the frustum.

Assume that three times of the difference between volume of the frustum of the cone and lateral surface area of the cylinder is equal to

$$
\pi\left[\mathrm{TO}_{h}+T_{76, h}-2 T_{6, h}-\text { Gno }_{h}+5\right]
$$

The mathematical statement of our assumption is

$$
\pi h\left[R^{2}+r^{2}+R r-6 r\right]=\pi h\left[16 h^{2}-12\right]
$$

This reduces to

$$
R^{2}+r^{2}+R r-6 r+12=16 h^{2}
$$

Introduce the linear transformation

$\mathrm{R}=\mathrm{u}+\mathrm{v} ; \mathrm{r}=\mathrm{u}-\mathrm{v}, u \neq 0, v \neq 0$

Therefore (2) reduces to

$3 x^{2}+y^{2}=z^{2}$

where $x=u-1 ; y=v+3 ; z=4 h$

We present below different methods of solving (4) and thus obtain different choices of non-zero integral values of the radii of the circular ends at the top and bottom of the frustum and the height of the cylinder cum frustum

\section{Pattern 1:}

Most cited solution to (4) is

$x=2 a b ; y=3 a^{2}-b^{2} ; z=3 a^{2}+b^{2}$

In view of (5) and (6)

$u=2 a b+1$

$v=3 a^{2}-b^{2}-3$

$h=\frac{3 a^{2}+b^{2}}{4}$

Since our aim is to find integer solution to height and radius, take $\mathrm{a}=2 \mathrm{~A} ; \mathrm{b}=2 \mathrm{~B}$.

Therefore we have,

$u=8 A B+1$

$v=12 A^{2}-4 B^{2}-3$

$h=3 A^{2}+B^{2}$

Using (8) and (9) in (3), the non-zero integral values of the radii of the circular ends at the top and bottom of the frustum and the height of the cylinder cum frustum satisfying (1) are given by

$$
\begin{aligned}
& R=12 A^{2}-4 B^{2}+8 A B-2 \\
& r=-12 A^{2}+4 B^{2}+8 A B+4
\end{aligned}
$$


Connections between Cylinder, Frustum of a Cone with Truncated Octahedral Number and Other Special Numbers

$h=3 A^{2}+B^{2}$

Some numerical examples satisfying our assumption are tabulated below

\begin{tabular}{|c|c|c|c|c|c|c|c|c|c|}
\hline \multicolumn{10}{|c|}{ Table1 } \\
\hline s.no & a & b & A & B & R & r & h & L.H.S of (1) & R.H.S of (1) \\
\hline 1 & 1 & 2 & 2 & 4 & 46 & 84 & 28 & 12544 & 12544 \\
\hline 2 & 2 & 2 & 4 & 4 & 254 & 4 & 64 & 65536 & 65536 \\
\hline 3 & 2 & 3 & 4 & 6 & 238 & 148 & 84 & 112896 & 112896 \\
\hline 4 & 3 & 4 & 6 & 8 & 558 & 212 & 172 & 473344 & 473344 \\
\hline 5 & 4 & 5 & 8 & 10 & 1006 & 276 & 292 & 1364224 & 1364224 \\
\hline
\end{tabular}

Pattern 2:

Let $\mathrm{z}=a^{2}+3 b^{2}$

Therefore (4) becomes $\left(a^{2}+3 b^{2}\right)^{2}=y^{2}+3 x^{2}$

Using factorization define

$[(a+i \sqrt{3} b)(a-i \sqrt{3} b)]^{2}=(y+i \sqrt{3} x)(y-i \sqrt{3} x)$

Equating real and imaginary we get

$x=2 a b ; y=a^{2}-3 b^{2}$

In view of (5) and (12)

$u=2 a b+1$

$v=a^{2}-3 b^{2}-3$

$h=\frac{a^{2}+3 b^{2}}{4}$

Since our aim is to find integer solution to height and radius, take

$\mathrm{a}=2 \mathrm{~A} ; \mathrm{b}=2 \mathrm{~B}$.

Therefore we have,

$u=8 A B+1$

$v=4 A^{2}-12 B^{2}-3$

$h=A^{2}+3 B^{2}$

Using (14) and (15) in (3), the non-zero integral values of the radii of the circular ends at the top and bottom of the frustum and the height of the cylinder cum frustum satisfying (1) are given by

$$
\begin{aligned}
& R=4 A^{2}-12 B^{2}+8 A B-2 \\
& r=-4 A^{2}+12 B^{2}+8 A B+4 \\
& h=A^{2}+3 B^{2}
\end{aligned}
$$


G.Janaki and P.Saranya

Some numerical examples satisfying our assumption are tabulated below.

\begin{tabular}{|c|c|c|c|c|c|c|c|c|c|}
\hline \multicolumn{10}{|c|}{ Table2 } \\
\hline s.no & a & b & A & B & R & r & h & L.H.S of $(1)$ & R.H.S of (1) \\
\hline 1 & 3 & 2 & 6 & 4 & 142 & 244 & 84 & 112896 & 112896 \\
\hline 2 & 6 & 4 & 12 & 8 & 574 & 964 & 336 & 1806336 & 1806336 \\
\hline 3 & 6 & 5 & 12 & 10 & 334 & 1588 & 444 & 3154176 & 3154176 \\
\hline 4 & 12 & 11 & 24 & 22 & 718 & 7732 & 2028 & 65804544 & 65804544 \\
\hline 5 & 13 & 9 & 26 & 18 & 2558 & 4932 & 1648 & 43454464 & 43454464 \\
\hline
\end{tabular}

Pattern 3:

Write (4) as

$$
z^{2}=1 .\left(y^{2}+3 x^{2}\right)
$$

Taking $\mathrm{z}$ as in (11), write $1=\frac{(1+i \sqrt{3})(1-i \sqrt{3})}{2^{2}}$

Using factorization define (17) as

$[(a+i \sqrt{3} b)(a-i \sqrt{3} b)]^{2}=\frac{(1+i \sqrt{3})(1-i \sqrt{3})}{2^{2}}[(y+i \sqrt{3} x)(y-i \sqrt{3} x)]$

Equating real and imaginary parts we have,

$$
\begin{aligned}
& x=\frac{1}{2}\left[a^{2}-3 b^{2}+2 a b\right] \\
& y=\frac{1}{2}\left[a^{2}-3 b^{2}-6 a b\right]
\end{aligned}
$$

In view of (5) and (18)

$$
\left.\begin{array}{l}
\begin{array}{l}
u=\frac{1}{2}\left[a^{2}-3 b^{2}+2 a b+2\right] \\
v
\end{array}=\frac{1}{2}\left[a^{2}-3 b^{2}-6 a b-6\right] \\
h=\frac{a^{2}+3 b^{2}}{4}
\end{array}\right\}
$$

Since our aim is to find integer solution to height and radius, take $a=2 A ; b=2 B$ Therefore we have,

$$
\left.\begin{array}{l}
u=2 A^{2}-6 B^{2}+4 A B+1 \\
v=2 A^{2}-6 B^{2}-12 A B-3
\end{array}\right\}
$$


Connections between Cylinder, Frustum of a Cone with Truncated Octahedral Number and Other Special Numbers

Using (20) in (3) with (21), the non-zero integral values of the radii of the circular ends at the top and bottom of the frustum and the height of the cylinder cum frustum satisfying (1) are given by

$$
\begin{aligned}
& R=4 A^{2}-12 B^{2}-8 A B-2 \\
& r=-16 A B+4 \\
& h=A^{2}+3 B^{2}
\end{aligned}
$$

Some numerical examples satisfying our assumption are tabulated below.

\begin{tabular}{|c|c|c|c|c|c|c|c|c|c|}
\hline \multicolumn{10}{|c|}{ Table 3} \\
\hline s.no & a & b & A & B & R & R & H & L.H.S of $(1)$ & R.H.S of $(1)$ \\
\hline 1 & 6 & 1 & 12 & 2 & 334 & 388 & 156 & 389376 & 389376 \\
\hline 2 & 7 & 1 & 14 & 2 & 510 & 452 & 208 & 692224 & 692224 \\
\hline 3 & 8 & 2 & 16 & 4 & 318 & 1028 & 304 & 1478656 & 1478656 \\
\hline 4 & 9 & 2 & 18 & 4 & 526 & 1156 & 372 & 2214144 & 2214144 \\
\hline 5 & 10 & 2 & 20 & 4 & 766 & 1284 & 448 & 3211264 & 3211264 \\
\hline
\end{tabular}

Pattern 4:

Write (4) as in (17)

Taking $\mathrm{z}$ as in (11) and write $1=\frac{(1+i 4 \sqrt{3})(1-i 4 \sqrt{3})}{7^{2}}$

Using factorization define (17) as

$[(a+i \sqrt{3} b)(a-i \sqrt{3} b)]^{2}=\frac{(1+i 4 \sqrt{3})(1-i 4 \sqrt{3})}{7^{2}}[(y+i \sqrt{3} x)(y-i \sqrt{3} x)]$

Equating real and imaginary parts we have

$$
\left.\begin{array}{l}
x=\frac{1}{7}\left[4 a^{2}-12 b^{2}+2 a b\right] \\
y=\frac{1}{7}\left[a^{2}-3 b^{2}-24 a b\right]
\end{array}\right\}
$$

In view of (5) and (22)

$$
\left.\begin{array}{l}
\begin{array}{l}
u=\frac{1}{7}\left[4 a^{2}-12 b^{2}+2 a b+7\right] \\
v
\end{array}=\frac{1}{7}\left[a^{2}-3 b^{2}-24 a b-21\right] \\
h=\frac{1}{4}\left[a^{2}+3 b^{2}\right]
\end{array}\right\}
$$

Since our aim is to find integer solution to height and radius, take $a=14 A ; b=14 B$. 
Therefore we have,

$$
\left.\begin{array}{l}
u=112 A^{2}-336 B^{2}+56 A B+1 \\
v=28 A^{2}-84 B^{2}-672 A B-3
\end{array}\right\}
$$

Using (24) in (3) with (25), the non-zero integral values of the radii of the circular ends at the top and bottom of the frustum and the height of the cylinder cum frustum satisfying (1) are given by

\begin{tabular}{|c|c|c|c|c|c|c|c|c|}
\hline \multicolumn{9}{|c|}{ Table4 } \\
\hline a & b & A & B & $\mathrm{R}$ & $\mathrm{r}$ & $\mathrm{h}$ & L.H.S of (1) & R.H.S of (1) \\
\hline 3 & & 49 & 7 & 1042719 & 4390400 & 1248520 & 24940835046400 & 24940835046400 \\
\hline 5 & 5 & 0 & 0 & 8 & 4 & 0 & 00 & 00 \\
\hline $\begin{array}{l}1 \\
2\end{array}$ & 2 & $\begin{array}{c}16 \\
8\end{array}$ & $\begin{array}{l}2 \\
8\end{array}$ & 724414 & 5597764 & 1498224 & 35914802466816 & 35914802466816 \\
\hline 3 & 4 & 54 & 5 & 2158430 & 4651080 & 1506867 & 36330399422876 & 36330399422876 \\
\hline 9 & & 6 & 6 & 2 & 4 & 6 & 20 & 20 \\
\hline $\begin{array}{l}4 \\
9\end{array}$ & 5 & $\begin{array}{c}68 \\
6 \\
\end{array}$ & $\begin{array}{l}7 \\
0\end{array}$ & $\begin{array}{c}3424511 \\
8\end{array}$ & $\begin{array}{c}7325382 \\
8\end{array}$ & $\begin{array}{c}2377950 \\
4\end{array}$ & $\begin{array}{c}90474369677762 \\
60\end{array}$ & $\begin{array}{c}90474369677762 \\
60\end{array}$ \\
\hline 7 & 1 & 98 & $\begin{array}{l}1 \\
4\end{array}$ & 417086 & 1756164 & 499408 & 3990533607424 & 3990533607424 \\
\hline
\end{tabular}

$$
\begin{aligned}
& R=140 A^{2}-420 B^{2}-616 A B-2 \\
& r=84 A^{2}-252 B^{2}+728 A B+4 \\
& h=49 A^{2}+147 B^{2}
\end{aligned}
$$

Some numerical examples satisfying our assumption are tabulated below.

\section{Conclusion}

In this paper, we expose connections between cylinder, frustum of a cone with truncated octahedral number and other special numbers and presented four patterns of solutions. In this approach, one may also search for various patterns of solutions and compare some other Geometrical figures with figurate numbers.

\section{REFERENCES}

1. L.E.Dickson, History of the theory numbers, Diophantine analysis New York, Dover, 2005.

2. W.Sierpinski, Pythagorean triangles, Dover publications, INC, Newyork, 2003.

3. R.D.Carmichael, The Theory of Numbers and Diophantine Analysis, Dover Publication, New York, 1959.

4. L.J.Mordell, Diophantine Equations, Academic press, London, 1969.

5. H.John, Conway, Richard K, Guy. The Book of Numbers, Springer Verlag, New York, 1995. 
Connections between Cylinder, Frustum of a Cone with Truncated Octahedral Number and Other Special Numbers

6. M.A.Gopalan and S.Devibala, On a Pythagorean problem, Acta Ciencia Indica, XXXII M (4) (2006) 1451-1452.

7. M.A.Gopalan and A.Gnanam, Pairs of Pythagorean triangles with equal perimeters, Impact. J. Sci Tech., 1(2) (2007) 67- 70.

8. M.A.Gopalan and S.Leelavathi, Pythagorean triangle with 2 area/perimeter as a cubic integer, Bulletin of Pure and Applied Science, 26 (2007) 197-200.

9. M.A.Gopalan and A.Gnanam, A special Pythagorean problem, Acta Ciencia Indica, XXXIII M (4) (2007) 1435- 1439.

10. M.A.Gopalan, A.Gnanam and G.Janaki, A remarkable pythagorean problem, Acta Ciencia Indica, XXXIII M (4) (2007) 1429-1434.

11. M.A.Gopalan and G.Janaki, Pythagorean triangle with area/perimeter as a special polygonal number, Bulletin of Pure and Applied Science, 27 (2008) 393-402.

12. M.A.Gopalan and A.Gnanam, Pythagorean triangles and Polygonal numbers, International Journal of Mathematical Sciences, 9(1-2) (2010) 211-215.

13. M.A.Gopalan and B.Sivakami, Pythagorean triangle with hypotenuse minus (area/perimeter) as a square integer, Archimedes. J Math., 2(2) (2012) 153-166.

14. M.A.Gopalan, Manjusomanath and K.Geetha, Pythagorean triangle with area/ perimeter as a special polygonal number, IOSR-JM, 7(3) (2013) 52-62.

15. G.Janaki and P.Saranya, Special pairs of pythagorean triangles and narcissistic number, International Journal of Multidisciplinary Research and Development, 3 (4) (2016) 106-108.

16. G.Janaki and P.Saranya, Special pythagorean triangles in connection with the narcissistic numbers of order 3 and 4, American International Journal of Research in Science, Technology, Engineering and Mathematics, 2(14) (2016) 150-153.

17. G.Janaki and P.Saranya, Special rectangles and narcissistic numbers of order 3 and 4, International Journal for Research in Applied Science and Engineering Technology, 4(VI) (2016) 630-633.

18. G.Janaki and P.Saranya, Special pairs of rectangles and narcissistic numbers of order 3 and 4, International Research Journal of Engineering and Technology, 3(8) (2016) 721-723.

19. G.Janaki and P.Saranya, Observations on truncated octahedral number, Universal Journal of Mathematics, 3(3) (2016) 87-92.

20. V.Pandichelvi and P. Sivakamasundari, Correlation of cylinder and frustum of the cone with special numbers, International Journal of Multidisciplinary Research and Development, 3(7) (2016) 241-242. 